\title{
Primordial magnetic helicity constraints from WMAP nine-year data
}

\author{
Tina Kahniashvili, ${ }^{1,2,3, *}$ Yurii Maravin, ${ }^{4,3, \dagger}$ George Lavrelashvili, ${ }^{5,6, \$}$ and Arthur Kosowsky ${ }^{7,8, \S}$ \\ ${ }^{1}$ McWilliams Center for Cosmology and Department of Physics, Carnegie Mellon University, \\ 5000 Forbes Avenue, Pittsburgh, Pennsylvania 15213, USA \\ ${ }^{2}$ Department of Physics, Laurentian University, Ramsey Lake Road, Sudbury, Ontario P3E 2C, Canada \\ ${ }^{3}$ Abastumani Astrophysical Observatory, Ilia State University, 3-5 Cholokashvili Street, \\ 0194 Tbilisi, Georgia \\ ${ }^{4}$ Department of Physics, Kansas State University, 116 Cardwell Hall, Manhattan, Kansas 66506, USA \\ ${ }^{5}$ Department of Theoretical Physics, A. Razmadze Mathematical Institute, \\ I. Javakhishvili Tbilisi State University, 0177 Tbilisi, Georgia \\ ${ }^{6}$ Max Planck Institute for Gravitational Physics, Albert Einstein Institute, 14476 Potsdam, Germany \\ ${ }^{7}$ Department of Physics and Astronomy, University of Pittsburgh, \\ 3941 O'Hara Street, Pittsburgh, Pennsylvania 15260, USA \\ ${ }^{8}$ Pittsburgh Particle Physics, Astrophysics, and Cosmology Center (Pitt-PACC), \\ Pittsburgh, Pennsylvania 15260, USA
}

(Received 6 August 2014; published 14 October 2014)

\begin{abstract}
If a primordial magnetic field in the Universe has nonzero helicity, the violation of parity symmetry results in nonzero correlations between cosmic microwave background temperature and B-mode polarization. In this paper we derive approximations to the relevant microwave background power spectra arising from a helical magnetic field. Using the cross-power spectrum between temperature and B-mode polarization from the WMAP nine-year data, we set a 95\% confidence level upper limit on the helicity amplitude to be $10 \mathrm{nG}^{2} \mathrm{Gpc}$ for helicity spectral index $n_{H}=-1.9$, for a cosmological magnetic field with effective field strength of $3 \mathrm{nG}$ and a power-law index $n_{B}=-2.9$ near the scale-invariant value. Future microwave background polarization maps with greater sensitivity will be able to detect the helicity of an inflationary magnetic field well below the maximum value allowed by microwave background constraints on the magnetic field amplitude.
\end{abstract}

DOI: $10.1103 /$ PhysRevD.90.083004

PACS numbers: $98.70 . V c, 98.80 .-\mathrm{k}$

\section{INTRODUCTION}

A challenging question of modern astrophysics is the origin of observed magnetic fields in galaxies and clusters [1]. Generally, fields observed today began as small seed fields and then were amplified via either adiabatic compression or through turbulent plasma dynamics. One mechanism for seed field generation is generic plasma instabilities and vorticity perturbations [2]. In this causal model, the correlation length of the resulting fields is limited by the horizon, which generically corresponds to comoving galaxy scales. A second possibility is larger seed fields generated during inflation spanning a wide range of correlation lengths up to the horizon today, and amplified through the process of cosmological structure growth $[3,4]$.

The evolution and amplification of a primordial seed field is strongly influenced by the helicity, or local handedness, of the seed field. Magnetic helicity is a manifestation of parity symmetry violation. While the level of parity violation observed in fundamental physical interactions is small,

\footnotetext{
tinatin@andrew.cmu.edu

†maravin@phys.ksu.edu

lavrela@itp.unibe.ch

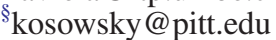

parity violation is widespread in various astrophysical systems with significant magnetic dynamics, such as onesided jets from active galactic nuclei and helical magnetic fields in the solar magnetosphere [5]. A seed field with helicity is restructured at large scales by plasma turbulence: the decay of the magnetic field leads to an increase in the relative magnetic helicity until the helicity saturates at the maximum value allowed by the realizability condition for the field strength. The magnetic field correlation length of a helical magnetic field will also increase more quickly than for a nonhelical field due to the inverse cascade mechanism. Magnetic fields with maximal helicity are a generic outcome of any extended period of turbulence [6].

Helical magnetic fields can be generated during the electroweak phase transition or during inflation [7-18]. Such a helical cosmological magnetic field might be the source of magnetic helicity needed in galactic dynamo amplification models [19]. Thus testing the helicity of any primordial magnetic field is important for understanding the origin of observed astrophysical magnetic fields [1]. Magnetic helicity in strong local magnetic fields like astrophysical jets can be deduced from the polarization of synchrotron radiation $[20,21]$. For cosmological magnetic helicity the detection issue is more difficult, because the field strengths are much lower and the observational effects more subtle. 
The most direct probe of any cosmological magnetic fields is their effect on the cosmic microwave background (CMB) radiation, and particularly its polarization. The microwave background linear polarization is conventionally decomposed into E-mode (parity-even) and B-mode (parity-odd) components [22,23]. A nonhelical magnetic field contributes to all of the parity-even power spectra, those correlating $\mathrm{E}$ with itself and B with itself, in addition to $\mathrm{E}$ with the microwave temperature $\mathrm{T}$ and the temperature with itself. These contributions were explicitly calculated in Ref. [24], and have been used to constrain the amplitude of a primordial magnetic field [25-32]. However, if a parityviolating helical magnetic field component is present, then it will contribute to the remaining parity-odd power spectra, namely EB or TB [33-36], which are identically zero for magnetic fields with zero helicity. Note that Faraday rotation by magnetic fields [37] imprints itself on the power spectrum and frequency spectrum of microwave background polarization, but is insensitive to helicity for a given magnetic field power spectrum [38-41].

Helical magnetic fields are perhaps the most natural parity-violating source of $\mathrm{TB}$ or $\mathrm{EB}$ correlations in the microwave background polarization [42-49], but other more speculative parity-violating sources can also induce them. These include a Chern-Simons coupling of photons to another field [42-44,50-52], a homogeneous magnetic field [53-56], Lorentz symmetry breaking [57-70], or nontrivial cosmological topology [71-74]. If some nonzero TB or EB correlation is detected, the corresponding angular power spectrum must be measured sufficiently well to distinguish between these possibilities.

In this paper we obtain upper limits on the helicity of a primordial magnetic field, using the nine-year WMAP constraints on any cross correlation between microwave background temperature and B-polarization [75]. Current polarization data are consistent with zero cosmological TB signal, as expected in the standard cosmological model. We compute the theoretical estimates of cross correlation given in Ref. [35] and compare with the measured upper limits [75-77]. Since we obtain only upper limits, we assume that magnetic helicity is the only possible parity-violating source present, which gives the most conservative helicity upper limits. For simplicity of calculation, we consider only the vector (vorticity) perturbations sourced by the magnetic field and neglect the tensor (gravitational wave) perturbations. This is a good approximation for angular multipoles $l>50$ [34], and for this reason we use measured $C_{l}^{\mathrm{TB}}$ constraints only for $l>50$; the neglected large angular scales contain little total statistical weight in our constraints.

The outline of the paper is as follows: in Sec. II we review the main characteristics of a helical magnetic field and derive the vorticity perturbations. Section III gives the expression for $C_{l}^{\mathrm{TB}}$ due to these vorticity perturbations, and these are compared with the WMAP nine-year upper limits in Sec. IV. Implications and future experimental prospects are discussed in Sec. V. We employ natural units with $\hbar=$ $c=1$ and Gaussian units for electromagnetic quantities.

\section{PROPERTIES OF A COSMOLOGICAL MAGNETIC FIELD}

We assume that a cosmological magnetic field was generated during or prior to the radiation-dominated epoch, with the energy density of the field being a first-order perturbation to the standard Friedmann-LemaitreRobertson-Walker homogeneous cosmological model. We also assume that primordial plasma is a perfect conductor and thus the spatial and temporal dependence of the field separates: $\mathbf{B}(\mathbf{x}, t)=\mathbf{B}(\mathbf{x}) / a(t)^{2}$ with $a(t)$ being the cosmological scale factor. The mean helicity density of the magnetic field is given by

$$
\mathcal{H}_{B}=\frac{1}{V} \int_{V} d \mathbf{x} \mathbf{A}(\mathbf{x}) \cdot \mathbf{B}(\mathbf{x})=\frac{1}{V} \int_{V} d \mathbf{x} \mathbf{A}(\mathbf{x}) \cdot \nabla \times \mathbf{A}(\mathbf{x}),
$$

with A being the vector potential, in the limit that the integral is over an infinite volume. An integral over a finite but large volume will approximate this helicity density. In general, magnetic helicity is a gauge-dependent quantity, because the vector potential $\mathbf{A}$ can be redefined by adding a gradient to it. However, the magnetic helicity is gauge invariant for periodic systems without a net magnetic flux, as shown in Ref. [78]. We assume that our Universe can be well approximated by a large box with periodic boundary conditions, provided the dimension of the box is large compared to the Hubble length today. In this case, the magnetic helicity is a well-defined quantity.

A Gaussian random magnetic field is described by the two-point correlation function in wave number space as

$$
\begin{aligned}
& \left\langle B_{m}^{*}(\mathbf{k}) B_{n}\left(\mathbf{k}^{\prime}\right)\right\rangle \\
& \quad=(2 \pi)^{3} \delta^{(3)}\left(\mathbf{k}-\mathbf{k}^{\prime}\right)\left[\left(\delta_{m n}-\hat{k}_{m} \hat{k}_{n}\right) P_{B}(k)+i \epsilon_{m n l} \hat{k}_{l} P_{H}(k)\right] .
\end{aligned}
$$

Here, $\hat{k}_{m}=k_{m} / k$ are the unit wave number components, $\epsilon_{m n l}$ is the antisymmetric tensor, and $\delta^{(3)}\left(\mathbf{k}-\mathbf{k}^{\prime}\right)$ is the Dirac delta function. We use the Fourier transform convention $B_{j}(\mathbf{k})=\int d^{3} x e^{i \mathbf{k} \cdot \mathbf{x}} B_{j}(\mathbf{x})$. The symmetric power spectrum $P_{B}(k)$ is related to the mean magnetic energy density by

$$
\mathcal{E}_{B}=\frac{1}{(2 \pi)^{3}} \int_{0}^{k_{D}} d k k^{2} P_{B}(k),
$$

while the antisymmetric power spectrum $P_{H}(k)$ is related to the magnetic helicity density as

$$
\mathcal{H}_{B}=\frac{1}{(2 \pi)^{3}} \int_{0}^{k_{D}} d k k \frac{1}{2} P_{H}(k)
$$


where $k_{D}$ is a characteristic damping scale for the magnetic field.

The total energy density and helicity of the magnetic field satisfy the realizability condition

$$
\mathcal{H}_{B} \leq 2 \xi_{M} \mathcal{E}_{B}
$$

where

$$
\xi_{M} \equiv \frac{2 \pi \int_{0}^{k_{D}} d k k P_{B}(k)}{\int_{0}^{k_{D}} d k k^{2} P_{B}(k)}
$$

is the magnetic field correlation length. The power spectra $P_{B}(k)$ and $P_{H}(k)$ are generically constrained by $P_{B}(k) \geq\left|P_{H}(k)\right|$. We assume that these power spectra are given by simple power laws, $P_{B}(k)=A_{B} k^{n_{B}}$ and $P_{H}(k)=A_{H} k^{n_{H}}$. The constraint on their relative amplitudes implies $n_{H}>n_{B}$ [79]; in addition, finiteness of the total magnetic field energy requires $n_{B}>-3$ if the power law extends to arbitrarily small values of $k$. For physical transparency, instead of describing the magnetic field amplitude by the proportionality factors $A_{B}$ and $A_{H}$, we will use the effective magnetic field amplitude $B_{\text {eff }} \equiv\left(8 \pi \mathcal{E}_{B}\right)^{1 / 2}[80]$ and the helicity density $\mathcal{H}_{B}$. Using these quantities is convenient because they do not depend on the power-law indices $n_{B}$ and $n_{H}$ and are independent of any smoothing scale.

Often, cosmological magnetic fields are characterized by a smoothed value on some comoving length scale $\lambda>\lambda_{D}=2 \pi / k_{D}$. Convolving with a Gaussian smoothing kernel, the smoothed magnetic field amplitude $B_{\lambda}$ is [24]

$$
\begin{aligned}
B_{\lambda}{ }^{2} & \equiv|\langle\mathbf{B}(\mathbf{x}) \cdot \mathbf{B}(\mathbf{x})\rangle|_{\lambda}=\frac{2}{(2 \pi)^{2}} A_{B} \Gamma\left(\frac{n_{B}+3}{2}\right) \lambda^{-n_{B}-3}, \\
\lambda & >\lambda_{D} .
\end{aligned}
$$

We also introduce a smoothed quantity $H_{\lambda}$ (the so-called helicity measure or current helicity [36]) related to the magnetic helicity having the same units as $B_{\lambda}$ and depending on the antisymmetric part of the magnetic field spectrum:

$$
\begin{aligned}
H_{\lambda}^{2} & \equiv \lambda|\langle\mathbf{B}(\mathbf{x}) \cdot[\nabla \times \mathbf{B}(\mathbf{x})]\rangle|_{\lambda} \\
& =\frac{2}{(2 \pi)^{2}} A_{H} \Gamma\left(\frac{n_{H}+4}{2}\right) \lambda^{-n_{H}-3}, \\
\lambda & >\lambda_{D} .
\end{aligned}
$$

See Ref. [40] for a more detailed discussion. Then the transformation between the smoothed quantities $B_{\lambda}$ and $H_{\lambda}$ and the effective quantities $B_{\text {eff }}$ and $\mathcal{H}_{B}$ is simply

and

$$
B_{\text {eff }}=\frac{B_{\lambda}\left(k_{D} \lambda\right)^{\frac{n_{B}+3}{2}}}{\sqrt{\Gamma\left(\frac{n_{B}+5}{2}\right)}},
$$

$$
\mathcal{H}_{B}=\frac{1}{8 \pi} \frac{\lambda H_{\lambda}^{2}\left(k_{D} \lambda\right)^{n_{H}+2}}{\left(n_{H}+2\right) \Gamma\left(\frac{n_{H}+4}{2}\right)}
$$

We assume that the magnetic field cutoff scale $k_{D}$ is determined by the Alfvén wave damping scale, $\lambda_{D} \simeq v_{A} L_{S}$ $[81,82]$, where $v_{A}$ is the Alfvén velocity set by the total magnetic energy density [24]. Since $v_{A} \ll 1$ the Alfvén damping scale will always be much smaller scale than the Silk damping scale (the thickness of the last scattering surface) for standard cosmological models. On the other hand, the CMB fluctuations are determined by the Silk damping scale, and presence of the magnetic field source at smaller scales will not significantly affect the resulting spectra.

\section{MICROWAVE BACKGROUND FLUCTUATIONS FROM A HELICAL MAGNETIC FIELD}

A cosmological magnetic field induces Alfvén waves sourced by the Lorentz force in the cosmological plasma (see [24,81-85]), which generically produce nonzero vorticity perturbations. In the case of a stochastic magnetic field the average Lorentz force $\langle\mathbf{L}(\mathbf{x})\rangle=-\langle\mathbf{B} \times[\nabla \times \mathbf{B}]\rangle /$ $(4 \pi)$ vanishes, while the root-mean-square Lorentz force $\langle\mathbf{L}(\mathbf{x}) \cdot \mathbf{L}(\mathbf{x})\rangle^{1 / 2}$ is nonzero and acts as a source in the vector perturbation equation. If the magnetic field spectrum Eq. (2) has a helical part $P_{H}(k)$, then the Lorentz force twopoint correlation function will have both symmetric and antisymmetric pieces. Both contribute to the symmetric piece of the vorticity perturbation spectrum, but only the antisymmetric piece of the Lorentz force, determined entirely by $P_{H}(k)$, will contribute to the antisymmetric part of the vorticity perturbation spectrum [35].

In the tight-coupling limit between photons and baryons, the fluid vorticity is sourced by the transverse and divergence-free piece of the Lorentz force. The fluid vorticity at last scattering then translates into temperature and polarization fluctuations in the microwave background radiation [24]. The microwave temperature and E-polarization components are both parity symmetric, while the B-polarization component is parity antisymmetric [86]. This implies that the cross-power spectra $C_{l}^{\mathrm{TB}}$ and $C_{l}^{\mathrm{EB}}$ from stochastic magnetic fields will be nonzero only if $P_{H}(k)$ is nonzero [22,33-36]. In other words, the TB and EB power spectra provide a way to measure whether a primordial magnetic field has a helical component. (A constant magnetic field component also gives nonzero $C_{l}^{\mathrm{TB}}$ and $C_{l}^{\mathrm{EB}}$ through Faraday rotation [53,54], but the two distinct contributions can be distinguished by their different power spectra, and by the frequency dependence of a Faraday rotation signal.)

Detailed computations of the various $\mathrm{CMB}$ angular power spectra induced by helical and nonhelical magnetic fields have been presented elsewhere [24,35]. Here we focus on the TB power spectrum, because current data do not put a significant constraint on the much smaller EB power spectrum. For $l>50$ where the TB power spectrum has significant power, we neglect tensor contributions, which are smaller. Here we derive an analytic approximation to the TB angular power spectrum, based on the 
second-order approximation technique from Ref. [87]; this approximate solution is simple and accurate enough for deriving upper limits on the helical magnetic field.

The multipoles of the temperature perturbation from a vector mode in Fourier space are given by

$\frac{\Theta_{l}^{( \pm 1)}\left(k, \eta_{0}\right)}{2 l+1} \simeq \sqrt{\frac{l(l+1)}{2}} \Omega^{( \pm 1)}\left(k, \eta_{\mathrm{dec}}\right) \frac{j_{l}\left(k \eta_{0}\right)}{k \eta_{0}}, \quad l \geq 2$

where $\Omega^{ \pm 1}(k, \eta)$ are the two helicity components of the gauge-invariant vorticity perturbations, constructed from the fluid velocity field and the vector component of the metric perturbations [35]. Here we have made the approximation $\eta_{0}-\eta_{\text {dec }} \simeq \eta_{0}$ in Eq. (11). For vorticity perturbations sourced by the magnetic field, the $l=1$ moment of temperature fluctuation is well approximated by the vorticity perturbation, $\Theta^{( \pm 1)}\left(k, \eta_{0}\right) \simeq \Omega^{ \pm 1}\left(k, \eta_{\mathrm{dec}}\right)$ [24]. For the B-mode polarization perturbation, we have [24]

$$
\begin{aligned}
\frac{B_{l}^{( \pm 1)}\left(k, \eta_{0}\right)}{2 l+1} \simeq & \mp \frac{\sqrt{6}}{2} \sqrt{(l-1)(l+2)} \\
& \times \int_{0}^{\eta_{0}} d \eta \dot{\tau}(\eta) e^{-\tau} P^{( \pm 1)}(k, \eta) \frac{j_{l}\left(k \eta_{0}-k \eta\right)}{k \eta_{0}-k \eta},
\end{aligned}
$$

where the polarization source is defined by [86]

$$
P^{( \pm 1)}=\frac{1}{10}\left[\Theta_{2}^{( \pm 1)}-\sqrt{6} E_{2}^{( \pm 1)}\right] .
$$

The temperature and polarization quadrupoles satisfy the evolution equations

$$
\begin{aligned}
& \dot{\Theta}_{2}^{( \pm 1)}=k\left[\frac{\sqrt{3}}{3} \Theta_{1}^{( \pm 1)}-\frac{2 \sqrt{2}}{7} \Theta_{3}^{( \pm 1)}\right]+\dot{\tau}\left[\Theta_{2}^{( \pm 1)}-P^{( \pm 1)}\right], \\
& \dot{E}_{2}^{( \pm 1)}=k\left[\mp \frac{1}{3} B_{2}^{( \pm 1)}-\frac{2 \sqrt{10}}{21} E_{3}^{( \pm 1)}\right]+\dot{\tau}\left[E_{2}^{( \pm 1)}+\sqrt{6} P^{( \pm 1)}\right] .
\end{aligned}
$$

Here the optical depth $\tau(\eta)=\int_{\eta}^{\eta_{0}} d \eta^{\prime} \dot{\tau}\left(\eta^{\prime}\right)$ to photon scattering from conformal time $\eta$ until today satisfies $d \tau / d \eta \equiv-\dot{\tau}(\eta)=\sigma_{T} n_{e}(\eta) a(\eta), \quad \sigma_{T}$ is the Thomson scattering cross section, and $n_{e}(\eta)$ the comoving number density of free electrons. The vector mode of the CMB temperature-B polarization angular power spectrum is given by [86]

$$
C_{l}^{\mathrm{TB}(V)}=\frac{2}{\pi} \int d k k^{2}\left[\frac{\Theta_{l}^{(-1) *}\left(k, \eta_{0}\right)}{2 l+1} \frac{B_{l}^{(-1)}\left(k, \eta_{0}\right)}{2 l+1}+\frac{\Theta_{l}^{(+1) *}\left(k, \eta_{0}\right)}{2 l+1} \frac{B_{l}^{(+1)}\left(k, \eta_{0}\right)}{2 l+1}\right] .
$$

In the rest of this section, we approximate this power spectrum in a given cosmological model, for comparison with limits on this power spectrum from temperature and polarization sky maps.

The visibility function $\dot{\tau} e^{-\tau}$ is sharply peaked at the time of decoupling, so to determine the B-polarization signal, Eq. (12), we need to know the polarization source $P^{( \pm 1)}$ at the time of decoupling. Differentiating Eq. (13) with respect to conformal time and substituting Eqs. (14) and (15), at leading order we get

$$
\dot{P}^{( \pm 1)}-\frac{3}{10} \dot{\tau} P^{( \pm 1)} \simeq \frac{k \sqrt{3}}{30} \Theta_{1}^{( \pm 1)}
$$

where we have dropped terms containing $\Theta_{3}^{( \pm 1)}, E_{3}^{( \pm 1)}$, and $B_{2}^{( \pm 1)}$ (see also Ref. [87]).

In our previous work [24,35], we assumed the first term of Eq. (17) is small to obtain the approximate solution $P^{( \pm 1)}=\sqrt{3} k \Theta_{1}^{( \pm 1)} / 9 \dot{\tau}$. While usually valid, this approximation fails during recombination because $k / \dot{\tau}$ varies rapidly: inserting $P^{( \pm 1)}=\sqrt{3} k \Theta_{1}^{( \pm 1)} / 9 \dot{\tau}$ into the integral of Eq. (12), the integrand becomes proportional to $e^{-\tau}$, and is not anymore peaked at the time of decoupling. Instead, we employ a more precise second-order approximate solution to the source equation, following the technique in Refs. $[87,88]$. Details are given in the appendix; the solution for the temperature-B polarization power spectrum is

$$
\begin{aligned}
C_{l}^{\mathrm{TB}} \simeq & -\frac{3 \pi}{14} \ln \left(\frac{10}{3}\right) \sqrt{\frac{(l+2) !}{(l-2) !}} \frac{\left(n_{B}+3\right)\left(n_{H}+2\right)}{n_{B}+n_{H}+2} \frac{\eta_{\mathrm{dec}}^{2}}{\eta_{0}^{2}} k_{D} \Delta \eta_{\mathrm{dec}} \frac{1}{\left(1+R_{\mathrm{dec}}\right)^{2}} \\
& \times \frac{\mathcal{E}_{B} \mathcal{H}_{B} k_{D}}{\rho_{\gamma 0}^{2}} \int_{0}^{x_{S}} d x x^{4} D_{E}(x)\left[1+\frac{n_{H}-1}{n_{B}+3} x^{n_{B}+n_{H}+2}\right] j_{l}^{2}\left(x k_{D} \eta_{0}\right)
\end{aligned}
$$


with the change of variables $x=k / k_{D}$ in the integral. We have defined a function which models the effect of Silk damping for polarization [88],

$$
D_{E}(x) \equiv 0.2\left(e^{-c_{E}\left(a_{1} x k_{D} \eta_{0}\right)^{b_{E}}}+e^{-c_{E}\left(a_{2} x k_{D} \eta_{0}\right)^{b_{E}}}\right)
$$

with the fitting constants $c_{E}=0.27, b_{E}=2.0$, $a_{1}=0.0011$, and $a_{2}=0.0019$. The amplitude of the approximate solution Eq. (18) differs from that in Ref. [24] by roughly a factor of two.

\section{CONSTRAINTS FROM WMAP}

We obtain constraints on primordial magnetic helicity by comparing the temperature-B-polarization cross correlation function in Eq. (18) with WMAP nine-year data. We assume a standard $\Lambda \mathrm{CDM}$ model. We take the Silk damping scale to be the thickness of the last scattering surface, $L_{S} \simeq \Delta \eta_{\mathrm{dec}}$, which is determined by the function $D_{E}(x)$, so $k_{S}=0.3 \mathrm{Mpc}^{-1}$. The WMAP $C_{l}^{\mathrm{TB}}$ measurement is consistent with a null signal, as expected in the standard cosmological model. We follow a Feldman-Cousins prescription [89] to set $68 \%$ and $95 \%$ confidence level upper limits on the primordial magnetic field [75]. We only consider multipoles with $\ell>50$ to simplify the analysis; in this range the measured values of $C_{\ell}^{\mathrm{TB}}$ are uncorrelated between different $\ell$ values. This restriction does not significantly impact sensitivity to the magnetic field, since most signal is for larger values of multipole number.

A comparison between our model for $C_{\ell}^{\mathrm{TB}}$ and the nineyear WMAP data is given in Fig. 1 for two magnetic field helicity models: one with power law $n_{H}=-1.9$ and amplitude $\mathcal{H}_{B}=10^{5} \mathrm{nG}^{2} \mathrm{Mpc}$, and one with power law $n_{H}=-0.6$ and amplitude $\mathcal{H}_{B}=10^{8} \mathrm{nG}^{2} \mathrm{Mpc}$. For both cases, we set the value of the effective magnetic field $B_{\text {eff }}$ to

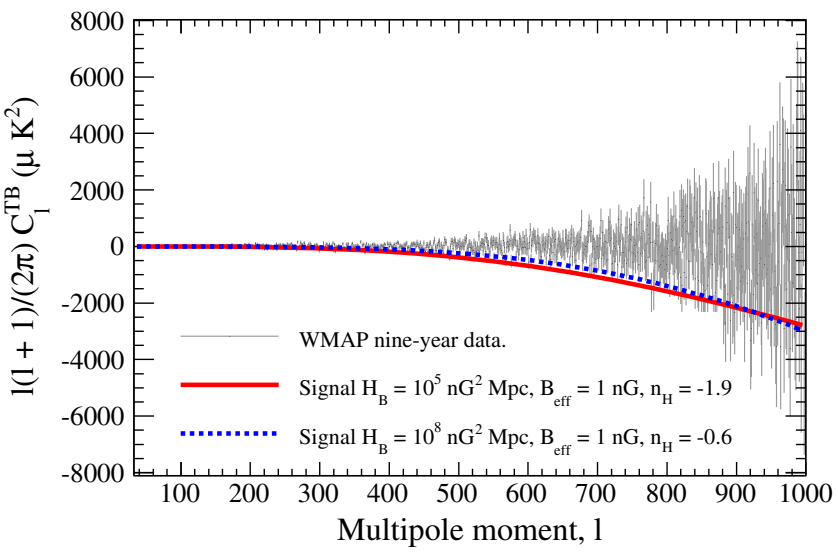

FIG. 1 (color online). A comparison between the temperatureB-polarization cross correlation model for $B_{\text {eff }}=1 \mathrm{nG}$, $n_{B}=-2.99$; the solid red line is for a helicity amplitude of $\mathcal{H}_{B}=$ $10^{5} \mathrm{nG}^{2} \mathrm{Mpc}$ and helicity spectral index $n_{H}=-1.9$ while the dotted blue line is for $\mathcal{H}_{\mathrm{B}}=10^{8} \mathrm{nG}^{2} \mathrm{Mpc}$ and $n_{H}=-0.6$. Also shown are the nine-year WMAP data (solid gray dots with bars indicating uncertainties).
$1 \mathrm{nG}$ and the spectral index to its inflationary value of $n_{B}=-2.99$, which is somewhat below current cosmological limits [32]. These models both produce a helical magnetic field which is just at the level which can be ruled out from the WMAP nine-year microwave background polarization power spectra. The helicity amplitude $\mathcal{H}_{B}$ varies strongly with spectral index, because for larger values of $n_{H}$ the helicity is more concentrated on small scales, close to the damping scale, which contribute little to the microwave background signal.

The upper limits on the $\mathcal{H}_{B}$ as functions of $n_{H}$ are given in Fig. 2 for three scenarios: $n_{B}=-2.99, n_{B}=-2.0$, and $n_{B}=n_{H}-1$. We also present the limits in terms of $H_{\lambda}$ for the same three scenarios in Fig. 3, using a smoothing scale of $\lambda=1 \mathrm{Mpc}$ which is commonly used in the magnetic field literature.

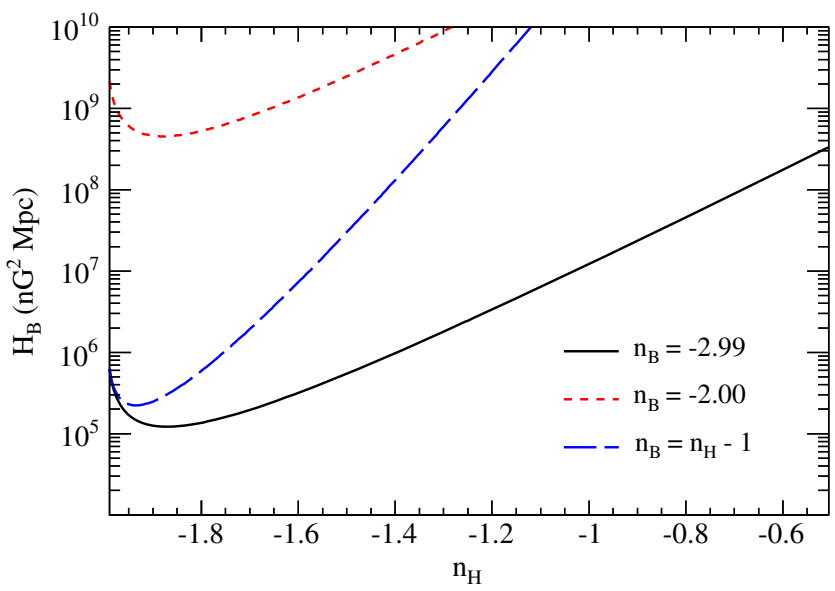

FIG. 2 (color online). The $95 \%$ upper limits on $\mathcal{H}_{B}$ as a function of $n_{H}$ for $n_{B}=-2.99$ (black solid), $n_{B}=-2.0$ (red short dash), and $n_{B}=n_{H}-1$ (blue long dash). For all three cases, $B_{\text {eff }}=1 \mathrm{nG}$.

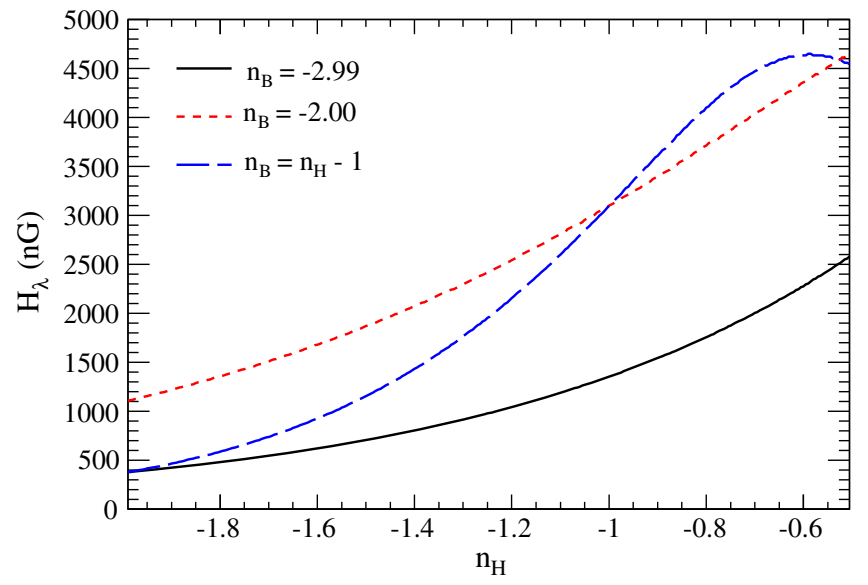

FIG. 3 (color online). The $95 \%$ upper limits on $\mathcal{H}_{\lambda}$ for a smoothing scale $\lambda=1 \mathrm{Mpc}$, as a function of $n_{H}$ for $n_{B}=-2.99$ (black solid), $n_{B}=-2.0$ (red short dash), and $n_{B}=n_{H}-1$ (blue long dash). For all three cases, $B_{\text {eff }}=1 \mathrm{nG}$. 
The results are relatively insensitive to the systematic uncertainty in the cross-correlation signal due to modeling of the cutoff scale; a plausible range of cutoff scales gives a signal difference which is smaller than the measurement uncertainties in the WMAP data. Systematic uncertainties with a size up to $20 \%$ of the predicted values of $C_{l}^{\mathrm{TB}}$ have only small effects on the magnetic field limits obtained here.

\section{CONCLUSIONS}

The results presented here are the first direct constraint on a helical primordial magnetic field by its contribution to the parity-odd temperature-B polarization cross-power spectrum $C_{l}^{\mathrm{TB}}$ of the microwave background. No experiment to date has detected a nonzero value for this power spectrum; we use the WMAP nine-year measurement which is consistent with zero to place upper limits on the combined mean field strength and helicity of a primordial magnetic field. The primordial magnetic field amplitude constraint of around $B_{\text {eff }}=3 \mathrm{nG}$ from the microwave background temperature and E-polarization power spectra [32,90-92] gives an upper limits on magnetic helicity $\mathcal{H}_{B}$ less than around $10 \mathrm{nG}^{2} \mathrm{Gpc}$ for a nearly scaleinvariant power spectrum with $n_{B}=-2.99$. The helicity limits become weaker for larger values of $n_{B}$. Recent work has argued for more stringent upper limits of $B_{\text {eff }}<1 \mathrm{nG}$ from constraints on the trispectrum induced by magnetic fields, rather than the power spectrum [93]. If magnetic fields from inflation are produced with a magnetic curvature mode as advocated by Ref. [94], then the trispectrum constraint is even stronger, pushing the magnetic field amplitude down to $B_{\text {eff }}<0.05 \mathrm{nG}$.

The mean helicity amplitude over a given volume is constrained by the realizability condition Eq. (5). The smaller the value of the magnetic field $B_{\text {eff }}$, the lower the helicity $\mathcal{H}_{B}$ which can be supported by the field. Any cosmological field will have physical effects measured over an effective volume which is at most the Hubble volume, so the effective comoving correlation length of this field is limited by the Hubble length $H_{0}^{-1}$. For a given microwave background constraint on $\mathcal{H}_{B}$ and assuming a magnetic field strength equal to some current upper limit, the maximal magnetic helicity which saturates the realizability condition must have a correlation length $\xi_{M}=4 \pi \mathcal{H}_{B} / B_{\text {eff }}^{2}$. If this correlation length is larger than the Hubble length, then a magnetic field of the given amplitude cannot support helicity as large as the measured limit. For a magnetic field with $B_{\text {eff }}=3 \mathrm{nG}$ and the corresponding helicity equal to the limiting value $\mathcal{H}_{B}=10 \mathrm{nG}^{2} \mathrm{Gpc}$, the correlation length for maximal helicity is around $10 \mathrm{Gpc}$ : current measurements provide a helicity constraint which is just at the level of the maximum possible helicity for the magnetic field strength. If the field strength is significantly lower, then the helicity limits derived in this paper are substantially above the maximum helicity allowed by Eq. (5).
Upcoming polarization data from the Planck satellite, as well as high-resolution ground-based experiments like ACTPol [95] and SPTPol [96], will strengthen limits on both the magnetic field amplitude and helicity, for two reasons: first, the signal increases for larger $l$ values beyond those probed by WMAP, and second, upcoming experiments will produce polarized maps over large portions of the sky with much greater sensitivity than WMAP. Interest in B-mode polarization has exploded due to the recent results from the BICEP2 collaboration [97]. Experiments searching for B-polarization from primordial tensor modes (at large angular scales) and gravitational lensing (at small angular scales) will drive continual increases in sensitivity over the coming decade. Planck's maps have a sensitivity (around $85 \mu \mathrm{K}$-arcmin for the SMICA map) which is a factor of 4 lower than WMAP (around $360 \mu \mathrm{K}$-arcmin), corresponding to errors in $C_{l}^{\mathrm{TB}}$ smaller by a factor of 16 . The recent PRISM satellite proposal [98] envisions full-sky polarization maps with sensitivity of $3 \mu \mathrm{K}$-arcmin, which would give $C_{l}^{\mathrm{TB}}$ errors smaller than the WMAP errors used here by a factor of $10^{4}$.

Limits on the magnetic field amplitude $B_{\text {eff }}$ from the microwave background power spectra will not improve substantially, because they are limited by cosmic variance in the power spectra from other nonmagnetic sources of fluctuations. In contrast, sensitivity improvements in polarization will continue to improve helicity limits from $C_{l}^{\mathrm{TB}}$ because this signal is not limited by cosmic variance: it is zero for standard-cosmology primary perturbations which do not violate parity. (At least this is the case until extreme sensitivities are reached where the cosmic variance in $C_{l}^{\mathrm{TB}}$ from the residual gravitational lensing contribution to delensed maps dominates over the map noise). So future measurements may provide constraints on magnetic field helicity which are much below the maximal helicity allowed by Eq. (5) and the magnetic field amplitude limits.

The TB power spectrum of cosmic microwave background polarization, and its lower-amplitude counterpart $\mathrm{EB}$, provide a valuable opportunity to probe unconventional physics which violates cosmological parity. Of contributors to these power spectra, gravitational lensing and helical magnetic fields are the two sources which rely only on standard, demonstrated physical effects. The microwave background lensing spectrum can be calculated to high accuracy within the standard model of cosmological structure formation, so any departures from this signal would be a good bet for revealing the existence of significant helical magnetic fields in the Universe. In turn, the detection of helicity would give valuable information about the stillmysterious origin of magnetic field in the cosmos.

\section{ACKNOWLEDGMENTS}

It is our pleasure to thank A. Brandenburg, L. Campanelli, K. Kunze, H. Tashiro, A. Tevzadze, and T. Vachaspati for useful discussions. T. K. and 
G. L. acknowledge partial support from the Swiss NSF SCOPES Grant No. IZ7370-152581. T. K. and A. K. were supported in part through NASA Astrophysics Theory program Grant No. NNX10AC85G and NSF Astrophysics and Astronomy Grant Program Grants No. AST-1109180 and No. AST-1108790. T. K. acknowledges partial support from Berkman Foundation. A. K. is partly supported by NSF Astrophysics and Astronomy
Grant No. AST-1312380. This work made use of the NASA Astrophysical Data System for bibliographic information.

\section{APPENDIX: DERIVATION OF $C_{l}^{\mathrm{TB}(V)}$}

The solution of Eq. (17) can be written in the form

$$
P^{( \pm 1)}=\frac{\sqrt{3}}{30} k \int_{0}^{\eta} d \eta^{\prime} e^{-\frac{3}{10} \tau\left(\eta, \eta^{\prime}\right)} \Theta_{1}^{( \pm 1)}\left(\eta^{\prime}\right)=\frac{\sqrt{3}}{30} k \int_{0}^{\eta} d \eta^{\prime} e^{+\frac{3}{10} \tau(\eta)} e^{-\frac{3}{10} \tau\left(\eta^{\prime}\right)} \Theta_{1}^{( \pm 1)}\left(\eta^{\prime}\right)
$$

where $\tau\left(\eta, \eta^{\prime}\right)=\int_{\eta^{\prime}}^{\eta} d \eta^{\prime \prime} \dot{\tau}\left(\eta^{\prime \prime}\right)=\tau\left(\eta^{\prime}\right)-\tau(\eta)$ and the visibility function $V(\eta)=\frac{d}{d \eta} e^{-\tau(\eta)}=\dot{\tau} e^{-\tau}$ can be approximated by the asymmetric Gaussian function $[88,99]$

$$
V(\eta)=V\left(\eta_{\mathrm{dec}}\right) \exp \left[-\frac{\left(\eta-\eta_{\mathrm{dec}}\right)^{2}}{2 \Delta \eta_{\mathrm{dec}}^{2}}\right]
$$

where $\Delta \eta_{\mathrm{dec}}=\Delta \eta_{\mathrm{dec}_{1}} \Theta\left(\eta_{\mathrm{dec}}-\eta\right)+\Delta \eta_{\mathrm{dec}_{2}} \Theta\left(\eta-\eta_{\mathrm{dec}}\right), \Delta \eta_{\mathrm{dec}_{1}}=0.0011 \eta_{0}$ and $\Delta \eta_{\mathrm{dec}_{2}}=0.0019 \eta_{0}$, and $\Theta(\eta)$ is the usual step function. The prefactor $V\left(\eta_{\mathrm{dec}}\right)=\frac{1}{\sqrt{2 \pi} \Delta \eta_{\mathrm{dec}}}$ is calculated from the normalization condition $\int_{0}^{\eta_{0}} V(\eta) d \eta=1$. Substituting the solution Eq. (A1) into Eq. (12) we obtain

$$
\frac{B_{l}^{ \pm 1}\left(k, \eta_{0}\right)}{2 l+1}=\mp \frac{k}{10 \sqrt{2}} \sqrt{(l-1)(l+2)} \int_{0}^{\eta_{0}} d \eta V(\eta) \frac{j_{l}\left(k\left(\eta_{0}-\eta\right)\right)}{k\left(\eta_{0}-\eta\right)} \int_{0}^{\eta} d \eta^{\prime} e^{+\frac{3}{10} \tau(\eta)} e^{-\frac{3}{10} \tau\left(\eta^{\prime}\right)} \Theta_{1}^{( \pm 1)}\left(\eta^{\prime}\right) .
$$

Since the visibility function $V(\eta)$ is sharply peaked around $\eta=\eta_{\mathrm{dec}}$ and $e^{-\frac{3}{10} \tau\left(\eta^{\prime}\right)}$ behaves like a step function, the $\Theta_{1}^{( \pm 1)}\left(\eta^{\prime}\right)$ factor can approximately be pulled out from the $\eta^{\prime}$ integration and we get

$$
\frac{B_{l}^{ \pm 1}\left(k, \eta_{0}\right)}{2 l+1}=\mp \frac{k}{10 \sqrt{2}} \sqrt{(l-1)(l+2)} \int_{0}^{\eta_{0}} d \eta V(\eta) \frac{j_{l}\left(k\left(\eta_{0}-\eta\right)\right)}{k\left(\eta_{0}-\eta\right)} \Theta_{1}^{( \pm 1)}(\eta) \int_{0}^{\eta} d \eta^{\prime} e^{+\frac{3}{10} \tau(\eta)} e^{-\frac{3}{10} \tau\left(\eta^{\prime}\right)} .
$$

Noticing that $V(\eta) \propto \exp \left(-\gamma\left(\eta-\eta_{\mathrm{dec}}\right)^{2}\right)$ and $j_{l}\left(k\left(\eta_{0}-\eta\right)\right)$ contains a mixture of oscillating modes $e^{i p \eta}$ and $e^{-i p \eta}$ with $p \propto k$, the formula $\int_{-\infty}^{\infty} e^{-\gamma \eta^{2}} e^{i p \eta} d \eta=e^{-p^{2} / 4 \gamma} \int_{-\infty}^{\infty} e^{-\gamma \eta^{2}} d \eta$ gives the approximation [88]

$$
\int_{0}^{\eta_{0}} d \eta V(\eta) \frac{j_{l}\left(k\left(\eta_{0}-\eta\right)\right)}{k\left(\eta_{0}-\eta\right)} \Theta_{1}^{( \pm 1)}(\eta) \approx \frac{j_{l}\left(k\left(\eta_{0}-\eta_{\mathrm{dec}}\right)\right.}{k \eta_{0}-k \eta_{\mathrm{dec}}} \Theta_{1}^{( \pm 1)}\left(\eta_{\mathrm{dec}}\right) D_{E}(k) \int_{0}^{\eta_{0}} d \eta V(\eta)
$$

where $D_{E}(k)$ is the Silk damping factor for polarization [88], Eq. (19).

Introducing a new variable $x \equiv \tau\left(\eta^{\prime}\right) / \tau(\eta)$, approximating $d \eta^{\prime}=-\Delta \eta_{\mathrm{dec}} d x / x$, and noticing that

$$
\int_{0}^{\eta_{0}} d \eta V(\eta) \int_{1}^{\infty} \frac{d x}{x} e^{-\frac{3}{10} x \tau(\eta)} e^{\frac{3}{10} \tau(\eta)}=\Delta \eta_{\operatorname{dec}} \cdot \int_{0}^{\infty} d \tau e^{-\frac{7}{10} \tau(\eta)} \int_{1}^{\infty} \frac{d x}{x} e^{-\frac{3}{10} x \tau(\eta)}=\Delta \eta_{\operatorname{dec}} \frac{10}{7} \ln \frac{10}{3},
$$

we get

$$
\begin{aligned}
\frac{B^{ \pm 1}\left(k, \eta_{0}\right)}{2 l+1} & =\mp \frac{\sqrt{2}}{14} \ln \left(\frac{10}{3}\right) \sqrt{(l-1)(l+2)} D_{E}(k) \frac{j_{l}\left(k \eta_{0}\right)}{k \eta_{0}} k \Delta \eta_{\mathrm{dec}} \Theta_{1}^{( \pm 1)}\left(k, \eta_{\mathrm{dec}}\right) \\
& =\mp \frac{\sqrt{2}}{14} \ln \left(\frac{10}{3}\right) \sqrt{(l-1)(l+2)} D_{E}(k) k \Delta \eta_{\mathrm{dec}} \frac{j_{l}\left(k \eta_{0}\right)}{k \eta_{0}} \Omega^{( \pm 1)}\left(k, \eta_{\mathrm{dec}}\right) .
\end{aligned}
$$

Making use of Eqs. (A8) and (11), we finally obtain for the temperature-B-polarization cross-correlation function 


$$
\begin{aligned}
C_{l}^{\mathrm{TB}(V)}= & -\frac{2}{7 \pi} \ln \left(\frac{10}{3}\right) \sqrt{\frac{(l+2) !}{(l-2) !}} \\
& \times \int d k k^{2} D_{E}(k) \omega\left(k, \eta_{\mathrm{dec}}\right) \frac{j_{l}^{2}\left(k \eta_{0}\right)}{\left(k \eta_{0}\right)^{2}} k \Delta \eta_{\mathrm{dec}}
\end{aligned}
$$

where $\omega(k)$ is the helical part of the power spectrum which can be expressed as [35]

$$
\omega(k, \eta)=\left[\frac{k \eta}{\left(\rho_{\gamma, 0}+p_{\gamma, 0}\right)\left(1+R_{\mathrm{dec}}\right)}\right]^{2} g(k) .
$$

Here $p_{\gamma 0}$ and $\rho_{\gamma 0}$ are the radiation pressure and energy density today, $R_{\mathrm{dec}}$ is the baryon-photon energy density at decoupling, and $g(k)$ can be expressed in terms of the spectral indices $n_{B}$ and $n_{H}$, values of $B_{\lambda}, H_{\lambda}$, and the smoothing scale $\lambda$ as

$$
g(k)=\mathcal{G} \lambda k\left(\lambda k_{D}\right)^{n_{B}+n_{H}+2}\left[1+\frac{n_{H}-1}{n_{B}+3}\left(\frac{k}{k_{D}}\right)^{n_{B}+n_{H}+2}\right]
$$

with

$$
\mathcal{G}=\frac{\lambda^{3} B_{\lambda}^{2} H_{\lambda}^{2}}{24\left(n_{B}+n_{H}+2\right) \Gamma\left(\frac{n_{B}+3}{2}\right) \Gamma\left(\frac{n_{H}+4}{2}\right)} .
$$

Then using Eqs. (A10), (A11), and (A12) in Eq. (A9) we arrive at Eq. (18).
[1] L. M. Widrow, Rev. Mod. Phys. 74, 775 (2002).

[2] R. M. Kulsrud and E. G. Zweibel, Rep. Prog. Phys. 71, 046901 (2008).

[3] A. Kandus, K. E. Kunze, and C. G. Tsagas, Phys. Rep. 505, 1 (2011).

[4] R. Durrer and A. Neronov, Astron. Astrophys. 21, 62 (2013).

[5] A. Brandenburg and K. Subramanian, Phys. Rep. 417, 1 (2005).

[6] A. G. Tevzadze, L. Kisslinger, A. Brandenburg, and T. Kahniashvili, Astrophys. J. 759, 54 (2012).

[7] J. M. Cornwall, Phys. Rev. D 56, 6146 (1997).

[8] M. Giovannini and M. E. Shaposhnikov, Phys. Rev. D 57, 2186 (1998).

[9] G. B. Field and S. M. Carroll, Phys. Rev. D 62, 103008 (2000).

[10] T. Vachaspati, Phys. Rev. Lett. 87, 251302 (2001).

[11] H. Tashiro, T. Vachaspati, and A. Vilenkin, Phys. Rev. D 86, 105033 (2012).

[12] G. Sigl, Phys. Rev. D 66, 123002 (2002).

[13] K. Subramanian and A. Brandenburg, Phys. Rev. Lett. 93, 205001 (2004).

[14] L. Campanelli and M. Giannotti, Phys. Rev. D 72, 123001 (2005).

[15] V. B. Semikoz and D. D. Sokoloff, Astron. Astrophys. 433, L53 (2005); V. B. Semikoz and D. D. Sokoloff, Int. J. Mod. Phys. D 14, 1839 (2005).

[16] A. Diaz-Gil, J. Garcia-Bellido, M. Garcia Perez, and A. Gonzalez-Arroyo, Phys. Rev. Lett. 100, 241301 (2008).

[17] L. Campanelli, Int. J. Mod. Phys. D 18, 1395 (2009).

[18] L. Campanelli, Phys. Rev. Lett. 111, 061301 (2013).

[19] R. Banerjee and K. Jedamzik, Phys. Rev. D 70, 123003 (2004).

[20] T. A. Ensslin, Astron. Astrophys. 401, 499 (2003).

[21] J. P. Vallée, New Astron. Rev. 48, 763 (2004).

[22] M. Kamionkowski, A. Kosowsky, and A. Stebbins, Phys. Rev. D 55, 7368 (1997).
[23] M. Zaldarriaga and U. Seljak, Phys. Rev. D 55, 1830 (1997).

[24] A. Mack, T. Kahniashvili, and A. Kosowsky, Phys. Rev. D 65, 123004 (2002).

[25] J. R. Shaw and A. Lewis, Phys. Rev. D 81, 043517 (2010).

[26] D. G. Yamazaki, K. Ichiki, T. Kajino, and G. J. Mathew, Adv. Astron. 2010, 586590 (2010).

[27] D. G. Yamazaki, K. Ichiki, T. Kajino, and G. J. Mathews, Phys. Rev. D 81, 103519 (2010).

[28] D. G. Yamazaki, K. Ichiki, T. Kajino, and G. J. Mathews, Phys. Rev. D 81, 023008 (2010).

[29] D. Paoletti and F. Finelli, Phys. Lett. B 726, 45 (2013).

[30] D. Paoletti and F. Finelli, Phys. Rev. D 83, 123533 (2011).

[31] K. E. Kunze, Phys. Rev. D 83, 023006 (2011).

[32] P. A. R. Ade et al. (Planck Collaboration), Astron. Astrophys., doi:10.1051/0004-6361/201321591 (2014).

[33] L. Pogosian, T. Vachaspati, and S. Winitzki, Phys. Rev. D 65, 083502 (2002).

[34] C. Caprini, R. Durrer, and T. Kahniashvili, Phys. Rev. D 69, 063006 (2004).

[35] T. Kahniashvili and B. Ratra, Phys. Rev. D 71, 103006 (2005).

[36] K. E. Kunze, Phys. Rev. D 85, 083004 (2012).

[37] A. Kosowsky and A. Loeb, Astrophys. J. 469, 1 (1996).

[38] T. A. Ensslin and C. Vogt, Astron. Astrophys. 401, 835 (2003).

[39] L. Campanelli, A. D. Dolgov, M. Giannotti, and F. L. Villante, Astrophys. J. 616, 1 (2004).

[40] A. Kosowsky, T. Kahniashvili, G. Lavrelashvili, and B. Ratra, Phys. Rev. D 71, 043006 (2005).

[41] T. Kahniashvili, Y. Maravin, and A. Kosowsky, Phys. Rev. D 80, 023009 (2009).

[42] P. Cabella, P. Natoli, and J. Silk, Phys. Rev. D 76, 123014 (2007).

[43] J. Q. Xia, H. Li, X. 1. Wang, and X. m. Zhang, Astron. Astrophys. 483, 715 (2008). 
[44] B. Feng, M. Li, J. Q. Xia, X. Chen, and X. Zhang, Phys. Rev. Lett. 96, 221302 (2006).

[45] J.-Q. Xia, J. Cosmol. Astropart. Phys. 01 (2012) 046.

[46] J.-Q. Xia, H. Li, and X. Zhang, Phys. Lett. B 687, 129 (2010).

[47] M. Li, Y.-F. Cai, X. Wang, and X. Zhang, Phys. Lett. B 680, 118 (2009).

[48] M. Li and X. Zhang, Phys. Rev. D 78, 103516 (2008).

[49] A. Gruppuso, P. Natoli, N. Mandolesi, A. De Rosa, F. Finelli, and F. Paci, J. Cosmol. Astropart. Phys. 02 (2012) 023.

[50] A. Lue, L. M. Wang, and M. Kamionkowski, Phys. Rev. Lett. 83, 1506 (1999).

[51] J. Q. Xia, H. Li, G. B. Zhao, and X. Zhang, Astrophys. J. 679, L61 (2008).

[52] S. Saito, K. Ichiki, and A. Taruya, J. Cosmol. Astropart. Phys. 09 (2007) 002.

[53] E. S. Scannapieco and P. G. Ferreira, Phys. Rev. D 56, R7493 (1997).

[54] C. Scoccola, D. Harari, and S. Mollerach, Phys. Rev. D 70, 063003 (2004).

[55] M. Demianski and A. G. Doroshkevich, Phys. Rev. D 75, 123517 (2007).

[56] J. R. Kristiansen and P. G. Ferreira, Phys. Rev. D 77, 123004 (2008).

[57] S. M. Carroll, G. B. Field, and R. Jackiw, Phys. Rev. D 41, 1231 (1990).

[58] V. A. Kostelecky and M. Mewes, Phys. Rev. Lett. 99, 011601 (2007).

[59] Y.-F. Cai, M. Li, and X. Zhang, J. Cosmol. Astropart. Phys. 01 (2010) 017.

[60] W.-T. Ni, Prog. Theor. Phys. Suppl. 172, 49 (2008).

[61] R. Casana, M. M. Ferreira, Jr., and J. S. Rodrigues, Phys. Rev. D 78, 125013 (2008).

[62] R. R. Caldwell, V. Gluscevic, and M. Kamionkowski, Phys. Rev. D 84, 043504 (2011).

[63] H. J. Mosquera Cuesta and G. Lambiase, J. Cosmol. Astropart. Phys. 03 (2011) 033.

[64] M. Kamionkowski and T. Souradeep, Phys. Rev. D 83, 027301 (2011).

[65] V. Gluscevic and M. Kamionkowski, Phys. Rev. D 81, 123529 (2010).

[66] M. Mewes, Phys. Rev. D 85, 116012 (2012).

[67] V. Gluscevic, D. Hanson, M. Kamionkowski, and C. M. Hirata, Phys. Rev. D 86, 103529 (2012).

[68] W.-T. Ni, in the 5th Patras Workshop on Axions, WIMPs, and WISPs, the University of Durham, 2009 (to be published).

[69] N. J. Miller, M. Shimon, and B. G. Keating, Phys. Rev. D 79, 103002 (2009).

[70] W.-T. Ni, Int. J. Mod. Phys. A 24, 3493 (2009).

[71] E. A. Lim, Phys. Rev. D 71, 063504 (2005).

[72] S. M. Carroll and E. A. Lim, Phys. Rev. D 70, 123525 (2004).
[73] S. H. S. Alexander, Phys. Lett. B 660, 444 (2008).

[74] M. Satoh, S. Kanno, and J. Soda, Phys. Rev. D 77, 023526 (2008).

[75] C. L. Bennett et al. (WMAP Collaboration), Astrophys. J. Suppl. Ser. 208, 20 (2013).

[76] G. Hinshaw et al. (WMAP Collaboration), Astrophys. J. Suppl. Ser. 208, 19 (2013).

[77] D. Larson, J. Dunkley, G. Hinshaw, E. Komatsu, M. R. Nolta, C. L. Bennett, B. Gold, M. Halpern et al., Astrophys. J. Suppl. Ser. 192, 16 (2011).

[78] M. A. Berger, Plasma Phys. Controlled Fusion 41, B167 (1999).

[79] R. Durrer and C. Caprini, J. Cosmol. Astropart. Phys. 11 (2003) 010.

[80] T. Kahniashvili, A. G. Tevzadze, and B. Ratra, Astrophys. J. 726, 78 (2011).

[81] K. Jedamzik, V. Katalinic, and A. V. Olinto, Phys. Rev. D 57, 3264 (1998).

[82] K. Subramanian and J. D. Barrow, Phys. Rev. Lett. 81, 3575 (1998).

[83] R. Durrer, T. Kahniashvili, and A. Yates, Phys. Rev. D 58, 123004 (1998).

[84] T. R. Seshadri and K. Subramanian, Phys. Rev. Lett. 87, 101301 (2001).

[85] K. Subramanian, T. R. Seshadri, and J. D. Barrow, Mon. Not. R. Astron. Soc. 344, L31 (2003).

[86] W. Hu and M. J. White, Phys. Rev. D 56, 596 (1997).

[87] M. Zaldarriaga and D. D. Harari, Phys. Rev. D 52, 3276 (1995).

[88] Z. Cai and Y. Zhang, Classical Quantum Gravity 29, 105009 (2012).

[89] G. J. Feldman and R. D. Cousins, Phys. Rev. D 57, 3873 (1998).

[90] J. R. Shaw and A. Lewis, Phys. Rev. D 86, 043510 (2012).

[91] D. G. Yamazaki, K. Ichiki, and K. Takahashi, Phys. Rev. D 88, 103011 (2013).

[92] T. Kahniashvili, Y. Maravin, A. Natarajan, N. Battaglia, and A. G. Tevzadze, Astrophys. J. 770, 47 (2013).

[93] P. Trivedi, K. Subramanian, and T. R. Seshadri, Phys. Rev. D 89, 043523 (2014).

[94] C. Bonvin, C. Caprini, and R. Durrer, Phys. Rev. D 88, 083515 (2013).

[95] M. Niemack et al., Proc. SPIE Int. Soc. Opt. Eng. 7741, $77411 \mathrm{~S}$ (2010).

[96] J. E. Austermann et al., Proc. SPIE Int. Soc. Opt. Eng. 8452, 84521E (2012).

[97] P. A. R. Ade et al., Phys. Rev. Lett. 112, 241101 (2014).

[98] P. Andre et al., arXiv:1306.2259.

[99] D.S. Gorbunov and V. A. Rubakov, Introduction To The Theory of The Early Universe: Cosmological Perturbations and Inflationary Theory (World Scientific, Singapore, 2011). 